the stepped care approach and community based rehabilitation model are relatively new initiatives.

It could be argued that all these models use the least possible healthcare resources. With fewer resources the community may be empowered to take on more responsibilities for ill people. Some may see this as a positive development, but others may see such initiatives as a way of transferring state responsibilities to the population. If there were to be such a diversification in provision of care in the developed world then it would be hoped that resources would follow responsibility.

The development of sufficient open access psychotherapeutic groups for depression as part of a stepped care approach, or the changes in tax and benefits required for increased support to new mothers, may only be cost neutral if they are balanced against the costs of poorly or untreated depression. If these initiatives represented a decrease in service quality or outcome, it would be difficult for developed countries to consider them.

In the developing world the poorest have no choice but to take what is offered. This is sometimes true of inner city areas in developed countries where novel cheaper healthcare initiatives are sometimes introduced. We understand the tension between value for money, innovation, and quality. We do not advocate the development of two tier healthcare systems in developed countries.

Of interest for developed countries is that when these simple models have been tested in the developing world they have been shown to be at least equally effective as more sophisticated and expensive ones. Moreover, whether through decreasing the cultural gulf between services and their recipients, increasing community involvement in health care, reinforcing preventive strategies, or developing more rational models of service delivery the examples that we have cited may offer added value.

Contributors: All authors contributed to the design, the writing, and the editing of this article. KMcK produced the first draft, taking into account the coauthors' comments.

Funding: KMcK and RA were supported by their universities. VP is supported by a Wellcome Trust career development fellowship in clinical tropical medicine.

Competing interest: None declared.

1 World Health Organization. The world health report 2001. Mental health: New understanding, new hope. Geneva: WHO, 2001.

Kleinman A Cohen A World mental health problems and priorities in low income countries. New York: Oxford University Press, 1995.

Becker AE, Lee DTS. Indigenous models for attenuation of post partum depression: case studies from Fiji and Hong Kong. In: Cohen A Kleinman A, Saraceno B, eds. World mental health casebook. New York: Kluwer, 2002

4 Lee DTS, Yip ASK, Leung TYS, Chung TKH. Ethnoepidemiology of postnatal depression: prospective multivariate study of sociocultural risk factors in a Chinese population in Hong Kong. Br J Psychiatry 2004;184:34-40.

5 MacArthur C, Winter HR, Bick DE, Lilford RJ, Lancashire RJ, Knowles H, et al. Re-designing postnatal care: a randomized controlled trial of protocol based midwifery-led care focused on individual women's physical and col based midwifery-led care focused on individual woments

6 Üstün TB, Ayuso-Mateos JL, Chatterji S, Mathers C, Murray JL. Global burden of depressive disorders in the year 2000. Br J Psychiatry

7 Araya R, Rojas G, Fritsch R, Gaete J, Rojas M, Simon G, et al. Treating depression in primary care in low income women in Santiago, Chile: a randomized controlled trial. Lancet 2003;361:995-1000.

8 Wells K, Sherbourne C, Schoenbaum M, Ettner S, Duan N, Miranda J, et al. Five-year impact of quality improvement for depression: results of a group-level randomized controlled trial. Arch Gen Psychiatry 2004:61: 378-86.

9 Guia Clinica para la Atencion Primaria: La Depresion: Deteccion, Diagnostico y Tratamiento. Programa de diagnostico y tratamiento integral de la depression en la atencion primaria. Santiago, Chile: Ministry of Health, 2009.

10 Hickling FW, McCallum M, Nooks L, Rodgers-Johnson P. Treatment of acute schizophrenia in open general medical wards in Jamaica. Psychiatr Serv 2000;51:659-63.

11 Chatterjee S, Patel V, Chatterjee A, Weiss H. Evaluation of a communitybased rehabilitation model for chronic schizophrenia in rural India. $\mathrm{Br}$ Psychiatry 2003;182:57-62.

\title{
Commentary: Community models of mental care warrant more governmental support
}

\author{
Malcolm W Battersby
}

Flinders Human Behaviour and Health Research Unit, Flinders University, Bedford Park, 5042, SA

Australia

Malcolm W Battersby senior lecturer in psychiatry

malcolm.battersby@ flinders.edu.au
Although developed countries are relatively well resourced there is an unmet demand for mental health services, particularly for anxiety disorders and depression. A similar and escalating demand exists for services for other forms of chronic illness too, such as diabetes secondary to obesity. In both, the potential of community care is increasingly recognised.

The core elements that emerge from the models McKenzie et al describe are community involvement in the planning and delivery of services, harnessing social support, and providers with a diversity of skill levels in stepped services. ${ }^{1}$ Replicating these models in wealthy countries would require overcoming considerable barriers in the form of system inertia, multiple funders, funding that creates disincentives to provide alternative services, professional vested interests and boundaries, stigma, loss of the sense of community, breakdown of the family, and lack of resources for the treatment and prevention of mental disorders compared with medical conditions.

But there are examples of services in developed countries that have overcome some of these barriers. The use of collaboratives as a large scale method of bringing about change in the delivery of services for chronic illness have been widely used in the United States, United Kingdom, and Canada and, more, recently Australia. ${ }^{2 \mathrm{w} 1}$ These collaboratives base their programme for change on the six elements of the chronic care model, ${ }^{3}$ one of which is support for self management. Although these programmes have improved health outcomes, they have focused on diabetes and heart failure rather than on mental illness. ${ }^{\mathrm{w} 2 \mathrm{w4}}$

The UK collaborative on falls prevention, which relies almost entirely on teams of community

References w1-w9 are on bmj.com 
members, takes this model a step further. ${ }^{4}$ It uses principles of social inclusion, social support, and team working in a "plan do study act" cycle as a way of delivering change at the community level. It suggests that empowered communities in deprived areas can achieve what health professionals alone are unlikely to achieve.

A model that shows that a health professional qualification is not necessary at all levels of service delivery is the peer led six week generic group programme for chronic illness self management developed at Stanford University. ${ }^{5}$ This programme has been adopted for widespread dissemination by the NHS as the expert patient programme ${ }^{w 5}$ Recent use of this model in a pilot programme for people with chronic severe mental illness in South Australia has shown positive outcomes. ${ }^{\text {w6 }}$

Stepped care for depression has been more efficacious than standard care in randomised trials in the United States, with primary care nurses supervised by a specialist providing case management by telephone. Similarly, a UK model for the provision of brief interventions in primary care has been developed. ${ }^{\mathrm{w} 7 \mathrm{w}}$ Elements of this approach have been transferred to South Africa in a mental health volunteer training programme. $^{\mathrm{w} 9}$
Adoption of programmes developed in a different setting requires a rigorous evaluation of their efficacy, effectiveness, and transferability. A more systematic evaluation might discern the key successful elements of these overseas programmes, something that is missing from McKenzie et al's article. Nevertheless, there is certainly no conceptual obstacle to the use of collaborative models for psychiatric disorders, and the models described should direct attention to determine what is transferable. They should also stimulate a look closer to home for examples in other areas of chronic illness care and a demand for the adoption of similar initiatives and resources from governments to address the crisis in prevention and management of mental disorders in developed countries.

Competing interests: None declared.

McKenzie K, Patel V, Araya R. Learning from low income countries: mental health. BMJ 2004;329:1138-40.

2 Institute for Health Improvement. The breakthrough series: IHI's collaborative model for achieving breakthrough improvement. Boston: IHI, 2003.

Wagner EH, Austin BT, Davis C, Hindmarsh M. Improving chronic illness care: translating evidence into action. Health Affairs 2001;20:64-78.

4 National Primary Care Development Team. Healthy communities collaborative-wave II www.npdt.org/scripts/defaultasp?site_id $=4$ (accessed 28 Oct 2004).

5 Lorig K, Ritter P, Stewart A, Sobel D, William Brown B, Bandura A, et al. Chronic disease self-management program: 2-year health status and health care utilization outcomes. Med Care 2201;39:1217-23.

\section{A memorable patient}

\section{The power of song}

Like most doctors, I have seen a great many deaths. Those in intensive care epitomise the undignified nature of death in modern Western society, but one becomes used to, inured to, fully monitored deaths, where coloured lines chart final dysfunction.

While working in Samoa, I helped to treat a middle aged patient. Two days after his admission to hospital with back pain, a diagnosis of dissecting thoracic aortic aneurysm was made, and he underwent surgery. The procedure was difficult and prolonged, with a large false lumen, no identifiable re-entry site, and probable carotid artery damage.

Within hours of arriving on the intensive care unit, he required an emergency thoracotomy for cardiac tamponade and developed evidence of incipient multiple organ failure. In the following discussion about treatment options, I was a firm advocate of quickly withdrawing treatment. However, surgical optimism overcame our pessimism, and he received increasingly heroic support with inotropes,

haemofiltration, ventilation, and (literally) gallons of blood products.

Throughout the first four days, his immediate family kept a vigil at his bedside. His wife spoke no English, and, despite translation, I was aware that none of our conversations was entirely successful. I felt that the fact her husband might die had been accepted, but little other information had crossed the barriers of language and culture.

On the fifth postoperative day, the patient's condition deteriorated again, and we finally convinced the surgeons that further treatment was futile. A priest was called, and the extended family, about two dozen in all, arrived. A sermon and invocation in Samoan was followed by an impromptu chorale, offering prayers and songs for the doomed. The juxtaposition of the rhythm of an intra-aortic balloon pump and melodious, reverberating psalm raised goose bumps on my skin. The family left, I switched off a dozen machines, pronounced death, and followed the family into the relatives' room, prepared to deliver "the usual spiel." Much to my surprise and embarrassment, I promptly burst into tears.

More than four days of translated talk, more than all the obvious high technology support we had provided, those tears broke the cultural barriers and showed the family what we had been trying to do. I was hugged, called brother, and comforted by those I had intended to comfort. Through my tears, I realised that I was providing the family with something positive, a more human face to Western medicine than perhaps they had previously encountered.

It was a strange and humbling experience, and has taught me much more than the fact that dying in intensive care does not have to be undignified. Few people in the West now die surrounded by so many friends and family as did this patient. As these things go, it was a good death. It has also, perhaps, refamiliarised me with the wider ramifications of death in intensive care.

Ian Nesbitt consultant in anaesthesia, Freeman Hospital, Newcastle upon Tyne (iannesbitt@yahoo.com)

We welcome articles up to 600 words on topics such as A memorable patient, A paper that changed my practice, $M y$ most unfortunate mistake, or any other piece conveying instruction, pathos, or humour. Please submit the article on http://submit.bmj.com Permission is needed from the patient or a relative if an identifiable patient is referred to. We also welcome contributions for "Endpieces," consisting of quotations of up to 80 words (but most are considerably shorter) from any source, ancient or modern, which have appealed to the reader. 